Прогностическое значение результатов инструментальных методов исследования при хронически протекающей ишемической болезни сердца. Данные регистра

\author{
Толпыгина С.Н., Марцевич С. Ю., Гофман Е.А., Аеев А.А. \\ ФГБУ “Государственный научно-исследовательский центр профилактической медицины” Минздрава \\ России. Москва, Россия
}

\begin{abstract}
Цель. Изучить роль результатов инструментальных методов исследования в оценке отдаленного прогноза жизни больных стабильно протекающей ишемической болезнью сердца (ИБС) в рамках регистра "ПРОГНОЗ ИБС".
\end{abstract}

Материал и методы. В рамках регистра проведено ретро-, проспективное, наблюдательное, когортное исследование, включившее всех пациентов, проживавших в Московском регионе, последовательно поступивших в стационар ГНИЦПМ с 01.01.2004 по 31.12.2007гг в плановом порядке с направительным диагнозом "ИБС", которым была выполнена коронароангиография. В исследование включен 641 пациент (500 мужчин и 141 женщина). Средний срок наблюдения - 3,9 года (0,76-6,52). Установлен жизненный статус 551 (86\%) пациента. В анализ были включены показатели инструментальных: электрокардиограмма (ЭКГ) покоя, эхокардиография (ЭхоКГ) и проба с дозированной физической нагрузкой (ПДФН) методов исследования.

Результаты. Риск развития первичной конечной точки (ПКТ) повышали: наличие на ЭКГ покоя рубцовых изменений в 2,2 раза ( $p=0,0007)$, тахикардии в 2,7 раза $(p=0,02)$, нарушений ритма в 1,76 раз $(\mathrm{p}=0,04) ;$ по данным ЭхоКГ - дилатация желудочков в 1,8 раз $(p=0,02)$, стенозы клапанов в 3 раза $(p=0,0015)$, диастолическая дисфункция в 2,5 раза ( $p=0,008)$, нарушение локальной сократимости в 1,8 раз $(p<0,003)$; развитие горизонтальной депрессии ST при ПДФН в 2,2 раза ( $p=0,04$, нд при кросстабуляции). Риск развития ПКТ снижали возможность выполнения ПДФН в 1,6 раз ( $p=0,049)$ и сохранная фракция выброса (ФВ) в 1,5 раз $(\mathrm{p}=0,001)$. Частоту развития вторичной комбинированной точки повышали: рубцовые изменения на ЭКГ в 1,5 раза ( $\mathrm{p}=0,01)$, положительный результат ПДФН на ишемию - увеличивал риск в 13,2 раз ( $p=0,01)$, косонисходящая депрессия сегмента ST при проведении ПДФН - в 2,1 раз $(p=0,01)$, низкая и средняя толерантность к физической нагрузке $2,8(p=0,04)$, и 2,1 раза $(p=0,04)$, соответственно. Наибольшее неблагоприятное влияние на прогноз по ПКТ оказали наличие рубцовых изменений на ЭКГ покоя, расширение полостей сердца, нарушение локальной сократимости левого желудочка, наличие стенозов клапанов по данным ЭхоКГ, а благоприятное - сохранная ФВ левого желудочка и факт проведения ПДФН.

Заключение. Высокая прогностическая значимость ряда данных широкодоступных инструментальных методов исследования, таких как ЭКГ покоя, ЭхоКГ и ПДФН на тредмиле, позволяет использовать их для стратификации риска развития сердечно-сосудистых осложнений у пациентов с хронической ИБС и определения показаний к проведению инвазивных диагностических процедур.

Ключевые слова: хроническая ишемическая болезнь сердца, регистр, инструментальные методы исследования, прогноз.

Кардиоваскулярная терапия и профилактика, 2014; 13 (4): 29-35

Поступила 21/04-2014

Принята к публикации 22/05-2014

\title{
Prognostic value of intstrumental diagnostics in chronic coronary heart disease. Data from "PROGNOS CHD"
}

Tolpygina S. N., Martsevich S. Yu., Gofman E. A., Deev A.D.

FSBI State Scientific-Research Centre for Preventive Medicine of the Ministry of Health, Moscow, Russia

Aim. To study the role of instrumental findings in the evaluation of longterm outcomes and life for stable coronary heart disease patients (CHD) in the "PROGNOS CHD" registry.

Material and methods. In the frame of registry the retro-, prospective, observational, cohort study is performed, that included all patients inhabitants of Moscow region, consequently admitted to the hospital of SSRCPM from 01.01.2004 to 31.12.2007 planned with the admittance dignosis "CHD", and for whom the coronary arteriography was done. Totally $641 \mathrm{pt}$ (500 male, 141 female). Mean follow-up period 3,9 years $(0,76-6,52)$. At the end the life status defined for 551 (86\%) patient. Into analysis the data from instrumental methods included: ECG, echocardiography (Echo), exercise testing (ET).

Results. The risk for primary endpoint (PEP) was increased by: scar changes in resting ECG $-2,2$ times $(p=0,0007)$, tachicardia 2,7 times $(p=0,02)$, rhythm disorders 1,76 times $(p=0,04)$; valve stenoses by Echo -3 times $(p=0,04)$, ventricle dilation 1,8 times $(p=0,02)$, local contractility defects 1,8 times $(p<0,003)$; ST horizontal depression during $E T-2,2$ times ( $p=0,04$, ns by crosstabulation). Risk of PEP decreased by the ET possibility to perform - by 1,6 times $(p=0,049)$, normal EF by 1,5 times $(p=0,001)$. The prevalence of secondary endpoint was increased by: scar changes on ECG $-1,5$ times $(p=0,01)$; positive ET $-13,2$ times $(p=0,01)$, downsloping ST depression at $E T-2,1$ times $(p=0,01)$, low and medium exercise tolerance $-2,8(p=0,04)$ and 2,1 times $(p=0,04)$, resp. The worse for PEP were scar changes on ECG, dilation of the heart chambers, disordered local contractility of LV, valve stenoses by Echo, and positively influenced outcomes - normal EF and the fact of ET performing.

\footnotetext{
*Автор, ответственный за переписку (Corresponding author):

Тел./факс: 8 (906) 793-92-63

e-mail: stolpygina@gnicpm.ru
}

[Толпыгина С. Н. ${ }^{\star}$ - к.м.н., вед.н. сотр. отдела профилактической фармакотерапии, Марцевич С. Ю.- д.м.н., проф., руководитель отдела, Гофман Е.А.- н.с. отдела, Деев А.Д.- к.ф.- м.н., руководитель лаборатории биостатистики] 
Conclusion. High predictive significance of various broad known instrumental methods of diagnostics, like resting ECG, Echo or ET, make possible to use them for risk stratification of cardiovascular complications development in chronic $\mathrm{CHD}$ and for defining of indications to invasive diagnostic procedures.
Key words: chronic ischemic heart disease, registry, instrumental diagnostics, prognosis.

Cardiovascular Therapy and Prevention, 2014; 13 (4): 29-35

АГ - артериальная гипертония, ВКТ - вторичная конечная точка, ДИ - доверительный интервал, ИБС - ишемическая болезнь сердца, КАГ - коронарная ангиография, лЖ - левый желудочек, ОР - относительный риск, ОС - общая смертность, ПДФН - проба с дозированной физической нагрузкой, ПКТ - первичная конечная точка, ССС - сердечно-сосудистое событие, ТТ - тредмил-тест, ТФН - толерантность к физической нагрузке, ФВ - фракция выброса, ХИБС - хроническая ишемическая болезнь сердца, ХСН - хроническая сердечная недостаточность, чСС - частота сердечных сокращений, ЭКГ электрокардиография, ЭхоКГ - эхокардиография, METs - metabolic equivalents, метаболические единицы.

\section{Введение}

Стратификация риска при хронической ишемической болезни сердца (ХИБС) является важнейшим этапом обследования таких больных. Её необходимость четко отражена во всех современных клинических рекомендациях, включая последние рекомендации Европейского общества кардиологов [1]. Для практической медицины крайне важен вопрос, какие методы являются необходимыми и достаточными для объективной оценки прогноза ХИБС у конкретного больного. Целью организованного регистра "ПРОГНОЗ ИБС" является изучение отделенных исходов у больных ХИБС, подтвержденной ангиографически, и выявление факторов, влияющих на исход заболевания. В предыдущих публикациях рассматривались значение клиникоанамнестических данных.

Цель настоящей публикации - изучить роль результатов ряда инструментальных методов исследования: электрокардиография (ЭКГ) покоя, эхокардиография (ЭхоКГ) и проба с физической нагрузкой (ПДФН) в оценке отдаленного прогноза жизни больных стабильно протекающей ИБС в рамках регистра “ПРОГНОЗ ИБС”.

\section{Материал и методы}

В регистр “ПРОГНОЗ ИБС” были включены пациенты, проживавшие в Московском регионе, последовательно поступавшие в плановом порядке в стационар ГНИЦПМ с 01.01.2004 по 31.12.2007гг с направительным диагнозом "ИБС" для обследования и лечения, и которым во время госпитализации была проведена коронарная ангиография (КАГ). Критериям отбора соответствовал 641 пациент. Средний срок наблюдения составил 3,9 года (от 0,76 до 6,52 лет). В 2010г устанавливали жизненный статус пациентов (жив, умер) и регистрировали конечные точки, развившиеся с момента выписки из стационара. Удалось установить судьбу 551 (86\%) больного.

Регистрация ЭКГ покоя в 12-отведениях в положении лежа выполнялась преимушественно на аппарате Schiller Cardiovit SC-200 на скорости 50 мм/мин во время повторного визита. Данные об аппарате, на котором проводилась регистрация ЭКГ во время госпитализации, отсутствуют.

Всего ПДФН - тредмил тест (ТТ) или велоэргометрия во время госпитализации была выполнена 330 пациентам, из них 260 на ТТ, которые и были включены в анализ прогностической значимости интегральных индексов, результаты которого представлены ранее [2, 3]. ТT выполняли на тредмиле на аппарате Schiller Cardiovit SC-200 пациентам с синусовым ритмом на ЭКГ, не имеющим противопоказаний к нему, по протоколу, разработанному в Центре, для научной и практической деятельности на “чистом фоне" или после отмены антиангинальной терапии за сут. до ТT, в соответствии с рекомендациями Всероссийского научного общества кардиологов [4].

Результаты ТТ оценивались на основании вариантов результатов ПДФН, предложенных Д.М. Ароновым и В. П. Лупановым [5].

Оценивали следующие показатели ПДФН:

- результат ПДФН: положительный, отрицательный, сомнительный, толерантность

- продолжительность нагрузки в мин и достигнутая мощность нагрузки в METs

- частота сердечных сокращений (ЧСС) до нагрузки и максимально достигнутая ЧСС

- выраженность стенокардии: отсутствие боли, боль не требовала прекращения $\Phi Н$, требовала прекращения $\Phi Н$, требовала приема нитроглицерина,

- смещение сегмента ST на высоте $Ф Н$, тип депрессии сегмента ST (горизонтальный, косонисходящий, косовосходящий), число отведений, в которых сместился сегмент ST

• причина прекращения ПДФН: прекращалась при достижении субмаксимальной ЧСС или развитии приступа стенокардии, требующего остановки $Ф Н$, а также при появлении других признаков или невозможность продолжать ПДФН по мнению больного.

ЭхоКГ выполняли в В- и М-режимах. При доплерЭхоКГ пользовались электронным векторным датчиком с частотой генератора ультразвуковых колебаний 2,53,5 МГц и с одновременной регистрацией стандартных отведений ЭКГ со скоростью 100 мм/сек. Визуализацию структур сердца производили из парастернального и верхушечного доступов по длинной и короткой осям сердца в положении пациента на левом боку. Размеры левого и правого предсердий определяли общепринятыми методами. Определяли размер полости левого желудочка (ЛЖ) в систолу и диастолу, толщину межжелудочковой перегородки и задней стенки ЛЖ в диастолу. Конечный диастолический и конечный систолический объемы ЛЖ, показатели насосной и сократительной функции ЛЖ: ударный объём,\% переднезаднего укорочения ЛЖ в систолу, фракция выброса (ФВ) ЛЖ (\%) рассчитывали по L. Teichholtz et al. [6]

Результаты инструментальных методов исследования сопоставляли с отдаленными исходами заболевания: Первичная комбинированная конечная точка (ПКТ) вклю- 
Прогностическая значимость данных ЭКГ покоя

\begin{tabular}{lllll}
\hline Признак & \multicolumn{2}{l}{ Риск развития ПКТ } & \multicolumn{2}{l}{ Риск развития ВКТ } \\
\cline { 2 - 5 } & $\mathrm{p}$ & $\mathrm{OP}($ ДИ 95\%) & $\mathrm{p}$ & ОР (ДИ 95\%) \\
\hline ЭКГ норма & 0,9867 & $1,012(0,214-4,203)$ & 0,3372 & $0,613(0,226-1,664)$ \\
\hline Синусовый ритм & 0,3191 & $0,680(0,319-1,452)$ & 0,8257 & $1,063(0,618-1,827)$ \\
\hline Тахикардия $>90$ уд/мин & 0,0234 & $2,670(1,142-6,241)$ & 0,4998 & $1,263(0,641-2,491)$ \\
\hline Брадикардия <55 уд/мин & 0,5809 & $0,839(0,450-1,564)$ & 0,7036 & $1,075(0,740-1,563)$ \\
\hline Рубцовые изменения на ЭКГ & 0,0007 & $2,237(1,402-3,568)$ & 0,0053 & $1,493(1,126-1,980)$ \\
\hline $\begin{array}{l}\text { Любые нарушения ритма сердца } \\
\text { на ЭКГ }\end{array}$ & 0,0356 & $1,761(1,039-2,985)$ & 0,2125 & $0,776(0,521-1,156)$ \\
\hline Изменения SТ & 0,418 & $1,355(0,650-2,827)$ & 0,1629 & $1,364(0,882-2,110)$ \\
\hline Атриовентрикулярная блокада & 0,8490 & $1,103(0,403-3,021)$ & 0,4136 & $0,730(0,343-1,551)$ \\
\hline Блокада левой ножки пучка Гиса & 0,0554 & $1,795(0,987-3,265)$ & 0,8972 & $0,971(0,617-1,527)$ \\
\hline
\end{tabular}

Примечание: 95\% ДИ - 95\% доверительный интервал.

чала случаи смерти от любых причин (ОС) и случаи нефатальных сердечно-сосудистых событий (ССС): инфаркт миокарда, мозговой инсульт/ транзиторная ишемическая атака (ИМ, МИ/ТИА); Вторичная комбинированная конечная точка (BKT) включала наряду со случаями смерти от любых причин и нефатальных ССС, проведение реваскуляризации любого сосудистого бассейна: аортокоронарное шунтирование, транслюминальная баллонная коронарная ангиопластика, каротидная эндартерэктомия, реваскуляризация артерий нижних конечностей, возобновление симптомов стенокардии, госпитализации по поводу ухудшения течения ИБС. Реваскуляризация учитывалась как ВКТ, если она выполнялась через $>6$ мес. после КАГ и выписки из стационара в связи с учащением приступов стенокардии. $O C$ не являлась самостоятельной конечной точкой в связи с небольшим количеством зарегистрированных случаев смерти.

Статистический анализ данных проводился в лаборатории биостатистики ГНИЦПМ с использованием системы SAS версия 6.12. Для тестирования прогностической значимости факторов риска и исходных клинических состояний применялись методы: t-тест Стьюдента, критерий Фишера, асимптотический критерий $\chi^{2}$ Вальда. Для определения прогностически значимых показателей применяли регрессионную модель пропорционального риска Кокса. Поскольку пол и возраст значимо влияют на прогноз ИБС, данные признаки независимо учитывались при анализе всех других переменных, т.е. значимость каждого из признаков оценивалась уже при вычитании значения влияния на прогноз пола и возраста. Поправка значимости на прогноз пола и возраста применялась во всех моделях.

\section{Результаты}

В настоящей работе представлены результаты анализа взаимосвязи данных инструментальных методов исследований (за исключением КАГ), пациентов при референсной госпитализации с частотой развития конечных точек. В анализ были включены данные 551 из 641 пациентов, у которых удалось установить жизненный статус, из них за период наблюдения умерли 50 человек. Судьбу 90 (14\%) пациентов выяснить не удалось. Среди пациентов с установленным жизненным статусом $(\mathrm{n}=551)$ было 432 (78\%) мужчин и 119 женщин. Возраст пациентов колебался от 27 до 88 лет, средний возраст среди мужчин составил $57,7 \pm 0,4$ лет, женщин - 60,3 $\pm 0,7$. Длительность наблюдения от 0,76 до 6,52 лет, в среднем 3,9 лет. Симптомы стенокардии имели $77 \%$ больных. Перенесли в прошлом ИМ 46\%, процедуры реваскуляризации - $15 \%$ пациентов. Страдали артериальной гипертонией (АГ) 79\%, сахарным диабетом - 13\%, ожирением $-27 \%$. Имели хроническую сердечную недостаточность (ХCH) 27\%, хроническую почечную недостаточность $-15 \%$, гиперлипидемии $85 \%$ больных.

Основные клинические исходы в период наблюдения. Из 551 умерли 50 человек (10 женщин

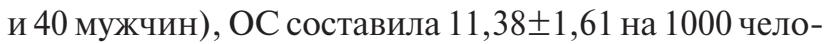
веко-лет. Наиболее частой причиной смерти были осложнения ХИБС (84\% от ОС). Процедуры реваскуляризации проведены после референсной госпитализации у 134 (24\%) больных. ПКТ развилась у 75 (14\%) больных, ВКТ у 198 (36\%).

Однофакторный анализ прогностической значимости признаков. В таблицах 1-3 представлены результаты однофакторного анализа прогностического значения изученных переменных в отношении риска развития ПКТ и ВКТ.

Достоверное отрицательное влияние на риск развития ПКТ оказали наличие рубцовых изменений на ЭКГ, нарушения ритма и тахикардия. Нарушения проводимости, изменения конечной части желудочкового комплекса не оказывали достоверного влияния на риск развития ПКТ. Наличие рубцовых изменений на ЭКГ увеличивало риск развития ВКТ в 1,5 раза (таблица 1). Остальные признаки на риск развития ВКТ в этом исследовании не повлияли.

Достоверное отрицательное влияние на относительный риск (ОР) развития ПКТ оказали сте- 
Прогностическая значимость данных ЭхоКГ

\begin{tabular}{lllll}
\hline Признак & \multicolumn{2}{l}{ Риск развития ПКТ } & \multicolumn{2}{l}{ Риск развития ВКТ } \\
\cline { 2 - 5 } & $\mathrm{p}$ & ОР (ДИ 95\%) & $\mathrm{p}$ & ОР (ДИ 95\%) \\
\hline Дилатация предсердий & 0,0528 & $1,591(0,994-2,546)$ & 0,8313 & $1,033(0,765-1,396)$ \\
\hline Дилатация желудочков & 0,0180 & $1,808(1,107-2,953)$ & 0,2611 & $1,205(0,871-1,667)$ \\
\hline Стеноз клапана по ЭхоКГ & 0,0015 & $2,950(1,511-5,761)$ & 0,5910 & $1,174(0,654-2,110)$ \\
\hline Недостаточность клапанов более 2 ст. & 0,1654 & $1,512(0,843-2,713)$ & 0,431 & $1,176(0,786-1,760)$ \\
\hline Нарушение локальной сократимости & 0,003 & $2,037(1,269-3,271)$ & 0,0094 & $1,461(1,098-1,946)$ \\
\hline Аневризма ЛЖ & 0,3668 & $1,47(0,637-3,392)$ & 0,1256 & $1,494(0,894-2,498)$ \\
\hline Гипертрофия ЛЖ & 0,2787 & $1,292(0,813-2,053)$ & 0,0860 & $1,284(0,965-1,709)$ \\
\hline Диастолическая дисфункция ЛЖ & 0,0079 & $2,472(1,268-4,820)$ & 0,007 & $1,903(1,311-2,761)$ \\
\hline Легочная гипертензия & 0,1537 & $1,777(0,806-3,918)$ & 0,6551 & $0,864(0,455-1,642)$ \\
\hline 1 квинтиль значения ФВ ЛЖ (18-41) & 0,1838 & $0,644(0,337-1,232)$ & 0,33556 & $1,226(0,810-1,856)$ \\
\hline 2 квинтиль значения ФВ ЛЖ (42-51\%) & 0,0546 & $0,530(0,277-1,013)$ & 0,5262 & $0,872(0,570-1,333)$ \\
\hline 3 квинтиль значения ФВ ЛЖ $(52-60 \%)$ & 0,008 & $0,292(0,118-0,725)$ & 0,0822 & $0,638(0,384-1,059)$ \\
\hline 4 квинтиль значения ФВ ЛЖ $(61-70 \%)$ & 0,1171 & $0,584(0,299-1,144)$ & 0,4282 & $0,833(0,530-1,310)$ \\
\hline 5 квинтиль значения ФВ ЛЖ $(>71 \%)$ & 0,4705 & $0,476(0,063-3,573)$ & 0,9892 & $0,993(0,354-2,787)$ \\
\hline
\end{tabular}

Таблица 3

Прогностическая значимость данных ПДФН (n=222)

\begin{tabular}{|c|c|c|c|c|}
\hline \multirow[t]{2}{*}{ Признак } & \multicolumn{2}{|c|}{ Риск развития ПКТ } & \multicolumn{2}{|c|}{ Риск развития ВКТ } \\
\hline & $\mathrm{p}$ & ОР (ДИ 95\%) & $\mathrm{p}$ & ОР (ДИ 95\%) \\
\hline Выполнение ТТ & 0,0493 & $0,607(0,369-0,998)$ & 0,1188 & $0,792(0,592-1,062)$ \\
\hline \multicolumn{5}{|c|}{ Результат ПДФН на наличие признаков ишемии миокарда } \\
\hline Отрицательный & нд & & 0,0745 & $6,402(0,832-49,255)$ \\
\hline Положительный & нд & & 0,0110 & $13,235(1,809-96,847)$ \\
\hline Сомнительный & нд & & 0,0631 & $6,685(0,902-49,577)$ \\
\hline $\begin{array}{l}\text { Оценка переносимости ФН на фоне } \\
\text { антиангинальной терапии }\end{array}$ & нд & & 0,0221 & $9,961(1,392-71,296)$ \\
\hline \multicolumn{5}{|l|}{ Выраженность боли } \\
\hline Не требовала прекращения ТТ & 0,2953 & $1,588(0,668-3,778)$ & 0,0046 & $2,029(1,244-3,308)$ \\
\hline Требовала прекращения ТТ & 0,2673 & $3,244(0,406-25,958)$ & 0,1681 & $2,763(0,651-11,724)$ \\
\hline Требовала приема нитроглицерина & 0,0339 & $2,153(1,060-4,371)$ & 0,0041 & $1,841(1,214-2,790)$ \\
\hline \multicolumn{5}{|l|}{ Количество отведений с депрессией ST } \\
\hline 3 & 0,1154 & $2,916(0,769-11,050)$ & 0,0180 & $2,463(1,167-5,197)$ \\
\hline 6 & 0,0092 & $3,889(1,400-10,80)$ & 0,0076 & $2,375(1,259-4,481)$ \\
\hline 10 & 0,0404 & $2,185(1,035-4,611)$ & 0,473 & $1,493(1,005-2,217)$ \\
\hline \multicolumn{5}{|l|}{ Тип депрессии ST } \\
\hline нет & 0,3374 & $1,803(0,541-6,013)$ & 0,9738 & $1,013(0,464-2,212)$ \\
\hline Косонисходящая & 0,1489 & $2,192(0,755-6,364)$ & 0,0107 & $2,148(1,194-3,864)$ \\
\hline Косовосходящая & 0,8548 & $1,119(0,335-3,735)$ & 0,7814 & $1,096(0,572-2,102)$ \\
\hline Горизонтальная & 0,0392 & $2,195(1,040-4,632)$ & 0,0460 & $1,496(1,007-2,222)$ \\
\hline \multicolumn{5}{|l|}{$\mathrm{T} \Phi \mathrm{H}$} \\
\hline Низкая ТФН (2,4-4,3 МЕТs) & 0,1011 & $2,352(0,846-6,537)$ & 0,0039 & $2,875(1,403-5,895)$ \\
\hline Средняя (4,3-7,0 METs) & 0,4302 & $1,612(0,492-5,283)$ & 0,0389 & $2,152(1,040-4,454)$ \\
\hline Высокая (7,0-14,2 METs) & 0,4750 & $1,538(0,472-5,015)$ & 0,039 & $2,590(1,356-4,9454,945)$ \\
\hline
\end{tabular}

нозы клапанов в 3 раза, нарушение локальной сократимости по ЭхоКГ в 2 раза, диастолическая дисфункция в 2,5 раза, дилатация желудочков в 1,8 раз. ФВ ЛЖ в диапазоне 52-60\% снижала риск развития ПКТ в 1,7 раз (таблица 2).
Достоверное отрицательное влияние на риск развития ВКТ из данных ЭхоКГ оказали лишь нарушение локальной сократимости (увеличение ОР в 1,5 раза) и диастолическая дисфункция (увеличение ОР в 1,9 раза). Для остальных признаков 
достоверного влияния на риск развития ВКТ не выявлено.

Достоверное отрицательное влияние на риск развития ПКТ оказали лишь небольшое число признаков, полученных при ТТ, - боль, возникшая во время ПДФН, потребовавшая приема нитратов, развитие горизонтальной депрессии ST и количество отведений $\geq 3$, в которых наблюдалась депрессия ST. Сам же факт выполнения ПДФН при ТТ снижал риск развития ПКТ в 1,4 раза (таблица 3 ).

Столь невысокое прогностическое значение данных ПДФН в отношении риска развития ПКТ обусловлено небольшим числом пациентов из тех, кому выполняли ПДФН, достигших ПКТ (всего 23 (16\%) из 222 больных и, следовательно, недостаточной статистической мощностью.

ВКТ достигли 39\% (71 из 222) больных, поэтому значения большего количества признаков оказались статистически значимы. Положительный результат ПДФН увеличивал риск развития ВКТ в 13,2 раз, развитие приступа стенокардии во время проведения ТТ, косонисходящая и горизонтальная депрессия сегмента ST при ПДФН в 2,1 и 1,5 раза, низкая ТФН ухудшала прогноз, достоверно увеличивая риск в 2,8 раза, соответственно. Отрицательное влияние на риск развития ВКТ средней и высокой ТФН при ТТ обусловлено, по-видимому, тем, что этим пациентам впоследствии проводились процедуры реваскуляризации в связи с наличием приступов стенокардии. Высокий ОР развития ВКТ (в 10 раз) у лиц, которым ПДФН выполнялась без отмены антиангинальной терапии, обусловлен тем, что диагноз ИБС у таких больных уже был верифицирован ранее, и целью проведения ПДФН была оценка переносимости ФН для решения вопроса о целесообразности назначения КАГ.

\section{Обсуждение}

Известно, прогноз ХИБС определяется тяжестью поражения коронарного русла [7-8], однако, учитывая невысокую доступность КАГ в России, сложность проведения и высокую стоимость, остается актуальным вопрос неинвазивной стратификации риска у пациентов с ИБС, доступной практическому врачу, в первую очередь, на основании клинических признаков и данных рутинных инструментальных исследований [1]. ОС является наиболее “жесткой” КT, однако для повышения мощности исследования в отношении определения прогноза были использованы ПКТ, которая включала помимо ОС, также случаи нефатальных ССС, и ВКТ, включавшая наряду со случаями ОС и нефатальных ССС, проведение реваскуляризации любого сосудистого бассейна, возобновление симптомов стенокардии, госпитализации по поводу ухудшения течения ИБС. По данным литературы с хорошим прогнозом ИБС ассоциируется нормальная ЭКГ покоя у пациентов с непродолжительным анамнезом заболевания и нормальной функцией ЛЖ [9]. В представленном исследовании (регистр “ПРОГНОЗ ИБС”) “нормальная" ЭКГ покоя не показала статистической значимости в отношении риска развития КТ, однако выявлено прогностическое значение наличия рубцовых изменений на ЭКГ покоя (достоверна для риска развития ПКТ в 2,2 и ВКТ в 1,5 раза) и тахикардии (>90 уд/мин) соответствует литературным данным $[10,11]$. Отрицательное влияние любых нарушений ритма на ЭКГ покоя также подтверждается данными многочисленных исследований о важном значении нарушений ритма сердца у пациентов с ИБС [12]. Таким образом, данные такого широкодоступного и рутинного метода исследования как ЭКГ покоя, имели высокую прогностическую значимость как в отношении риска развития ПКТ (рубцовые изменения, тахикардия и нарушения ритма сердца), так и ВКТ сердца (рубцовые изменения).

Известно, что одним из самых мощных предикторов выживаемости у больных ХИБС в отдаленные сроки является сократительная функция ЛЖ [1]. Сохранная функция ЛЖ относится к прогностическим факторам низкого риска (сердечно-сосудистая смертность $<1 \%$ в год), а сниженная функция ЛЖ (ФВ ЛЖ <50\%) относится к факторам высокого риска (расчетная ежегодная сердечно-сосудистая смертность >2\%) [13]. Известно, что прогноз у больных с хронической систолической дисфункцией ЛЖ ишемического происхождения неблагоприятный, несмотря на возможности современных методов лечения [8]. Значение ФВ ЛЖ по данным ЭхоКГ или рентгенографии является одним из наиболее мощных предикторов ССС [9, 14]. Доказано, что у больных стабильной стенокардией ОС увеличивается по мере снижения ФВ ЛЖ. При ФВ в покое $<35 \%$ ежегодная ОС $>3 \%$ [10]. В настоящем исследовании благоприятное влияние на прогноз оказала сохранная ФВ ЛЖ >51\% (снижение риска ПКТ на 70\%). Расширение полостей сердца повышало риск смерти в 2,6-3 раза, что согласуется с литературным данным, согласно которым, размеры желудочков сердца имеют прогностическое значение, превосходящее значение ПДФН.

Нарушение локальной сократимости ЛЖ по данным ЭхоКГ повышало риск развития ПКТ в 2 раза. По данным литературы также имеет значение объем инфарктного поражения (чем больше размер поражения, тем ниже сократительная способность миокарда, тем хуже прогноз у больного) [8]. Выявлено отрицательное влияние на прогноз наличие стенозов клапанов, в первую очередь аортального, (повышение риска ПКТ в 3 раза). Как известно, наличие симптомов стенокардии при аортальном стенозе ухудшает прогноз жизни больного, и требует проведения реваскуляризации миокарда до или во время протезирования аортального клапана. 
Отрицательное влияние нарушения диастолической функции ЛЖ на частоту развития ПКТ и ВКТ служит подтверждением важности этого параметра не только для развития симптомов ХCH, но и эффективности коронарного кровотока, происходящего именно в диастолу. Вероятно, прогностическое значение тахикардии на ЭКГ покоя имеет схожее объяснение, т.к. длительность фазы диастолы зависит от ЧСС. В течение длительного времени прогноз пациентов с ХСН связывали только с показателями систолической дисфункции. Однако низкие сократимость и ФВ не всегда определяют тяжесть декомпенсации, ТФН и даже прогноз больных ИБС, осложненной ХСН. В настоящее время получены убедительные доказательства того, что показатели диастолической функции в большей степени, чем снижение сократимости, коррелируют с клиническими и инструментальными маркерами декомпенсации, и могут быть использованы как надежные гемодинамические параметры для оценки эффективности лечения больного ИБС [15]. Патофизиологически диастола "чувствительна" к ишемии, еe расстройство может раньше и точнее всех других признаков свидетельствовать о вовлечении миокарда в патологический процесс. Современная концепция патогенеза ХСН при постинфарктном ремоделировании сердца рассматривается как совокупность изменения геометрии и объемов ЛЖ, гипертрофии миокарда, систолической и диастолической дисфункций ЛЖ. Снижение контрактильной способности миокарда в большинстве случаев сопровождается хотя бы минимальными нарушениями диастолической функции ЛЖ. В ряде случаев диастолическая дисфункция сердца может опережать развитие систолической дисфункции ЛЖ и изолированно приводить к развитию симптомов ХСН. Как показал результат анализа регистра "ПРОГНОЗ ИБС”, данные ЭхоКГ, второго после ЭКГ по доступности метода исследования, не имеющего противопоказаний к проведению, имеют высокую прогностическую значимость в отношении риска смерти и развития фатальных и нефатальных ССС и необходимости в повторных госпитализациях и процедурах реваскуляризации. Основное значение имели признаки поражения клапанного аппарата сердца, показатели сократительной функции миокарда ЛЖ (сохранность глобальной и нарушение локальной сократимости) и диастолической дисфункции. Характерно, что относительный риск развития ПКТ для признаков перенесенного ИМ по данным ЭхоКГ и ЭКГ оказались практически идентичными $(2,0$ и 2,2$)$. Увеличение риска отмечалось при наличии стенозов клапанов, диастолической дисфункции и расширения полостей сердца. Таким образом, проведение ЭхоКГ пациентам с предполагаемой или подтвержденной ИБС, позволяет более точно прогнозировать риск развития ССО.
Известно, что на прогноз при ИБС также влияет выраженность ишемии миокарда, которая может быть оценена при ПДФН. Высокая ТФН относится к прогностическим факторам низкого риска - сердечно-сосудистая смертность $<1 \%$ в год, а низкая ТФН - к факторам высокого риска (расчетная ежегодная сердечно-сосудистая смертность >2\%) [16]. В исследовании благоприятно влияла на прогноз возможность проведения ПДФН (снижение ПКТ на 40\%), что, по-видимому, было обусловлено менее тяжелым состоянием пациентов, поскольку в регистре “ПРОГНОЗ ИБС” ПДФН выполнялась преимущественно с диагностической целью, а пациенты с уже подтвержденным ранее диагнозом ИБС или имеющие типичную клинику стенокардии и сниженную ФВ ЛЖ, или перенесшие ИМ, согласно клиническим рекомендациям [1] направлялись на КАГ без предварительного проведения ПДФН. Выявленное достоверное влияние на частоту развития ПКТ лишь небольшого числа параметров, полученных при ТТ, обусловлено малым числом пациентов (16\%), которым выполнялась ПДФН, достигших ПКТ, и, следовательно, недостаточной статистической мощностью. Поскольку ВКТ достигли 39\% больных (преимущественно за счет процедур реваскуляризации), значение большего количества признаков оказалось статистически значимыми.

Ухудшали прогноз “положительный” результат пробы на ишемию (увеличение риска ВКТ в 13 раз), низкая ТФН (увеличение риска ВКТ в 9,9 раз) и такие ЭКГ-признаки ишемии миокарда, как косонисходящая и горизонтальная депрессия сегмента ST, регистрируемая в процессе ПДФН (увеличение риска развития ВКТ в 2 и 1,5 раза, соответственно) и количество отведений с депрессией сегмента ST $>3$, характеризующие тяжесть и обширность ишемии. Согласно литературным данным, прогностическими факторами являются переносимость ФН и клинические и ЭКГ-признаки ишемии миокарда, возникающие при ПДФН [16]. Выявление признаков ишемии миокарда на ЭКГ, в первую очередь в виде "ишемической” депрессии сегмента ST, с высокой вероятностью свидетельствует о неблагоприятном прогнозе. Выраженность этой ишемии находится в прямой связи с прогнозом заболевания. Также было показано, что у больных ИБС с нормальной или незначительно сниженной функцией ЛЖ 5-летняя выживаемость выше при более высокой ТФН [16].

Суммарный анализ показал, что из результатов инструментальных исследований наибольшее отрицательное влияние на прогноз имели: наличие рубцовых изменений, тахикардии и нарушения ритма сердца на ЭКГ покоя, нарушение локальной сократимости ЛЖ, расширение полостей желудочков сердца, наличие стенозов клапанов и диастолическая дисфункция по данным ЭхоКГ. Результаты ПДФН имели меньшее прогностическое значение в отноше- 
нии риска развития ПКТ, т.к. ПДФН выполнялась менее чем половине больных, причем наименее тяжелых. На риск развития ВКТ, включающий потребность в процедурах реваскуляризации, оказали “положительный” результат пробы на ишемию, низкая ТФН и косонисходящая и горизонтальная депрессия сегмента ST, отражающие выраженность ишемии. Отрицательное влияние на риск развития ВКТ средней и высокой ТФН при ТТ обусловлено, по-видимому, тем, что этим пациентам впоследствии проводились процедуры реваскуляризации в связи с наличием приступов стенокардии.

Таким образом, данные рутинной ЭКГ покоя имеют прогностическое значение для всех больных ИБС, ЭхоКГ позволяет более точно стратифицировать риск развития ССС, а проведение ПДФН целесообразно с диагностической целью и для отбора пациентов, имеющих промежуточный риск по кли-

\section{Литература}

1. 2013 ESC guidelines on the management of stable coronary artery disease. The Task Force on the management of stable coronary artery disease of the European Society of Cardiology. Eur Heart J 2013;34: 2949-3003 - doi:10.1093/eurheartj/ eht296.

2. Martsevich SYu, Tolpygina SN, Malysheva AM, et al. Role of selected parameters and integral indices of treadmill test in the assessment of complication risk among patients with chronic coronary heart disease. Cardiovascular Therapy and Prevention 2012; 11 (2): 44-52. Russian (Марцевич С. Ю., Толпыгина С. Н., Малышева А. М. др. Роль отдельных показателей и интегральных показателей и интегральных индексов пробы с дозированной физической нагрузкой на тредмиле в оценке риска осложнений у пациентов с хронической ишемической болезнью сердца. Кардиоваскулярная терапия и профилактика 2012; 11 (2): 44-52).

3. Martsevich SYu, Tolpygina SN, Malysheva AM, et al. Role of selected parameters and integral indices of treadmill test in the assessment of seriousness of coronary arteries stenosis among patients with chronic coronary heart disease. Cardiovascular Therapy and Prevention 2013; 12 (5): 22-8. Russian (Марцевич С. Ю., Толпыгина С.Н., Малышева А. М. и др. Значение отдельных показателей и интегральных показателей и интегральных индексов пробы с дозированной физической нагрузкой на тредмиле для выявления тяжести стенотического поражения коронарных артерий. Кардиоваскулярная терапия и профилактика 2013; 12 (5): 22-8).

4. Committee of experts of VNOK. Diagnostics and treatment of stable stenocardia Russian references (the second revision). Cardiovascular Therapy and Prevention 2008; 7 (6); Appendix 4: 40. Russian (Комитет экспертов ВНОК. Диагностика и лечение стабильной стенокардии. Российские рекомендации (второй пересмотр). Кардиоваскулярная терапия и профилактика 2008; 7 (6); Приложение 4: 40)

5. Aronov DM, Lupanov VP. Functional assays in a cardiology. Third edition. Moscow. "Medical press inform" 2007; 328 p. Russian (Аронов Д.М., Лупанов В.П. Функциональные пробы в кардиологии. Третье издание. Москва "МЕДпресс-информ" 2007; 328 с).

6. Teichholz LE, Kreulen T, Herman MV, et al. Problems in echocardiographic volume determinations: echocardiographic-angiographic correlations in the presence of absence of asynergy. Am J Cardiol 1976; 37 (1): 7-11. нико-анамнестическим данным, результатам ЭКГ покоя и ЭхоКГ, для проведения КАГ и процедур реваскуляризации.

\section{Заключение}

В регистре “ПРОГНОЗ ИБС” подтверждена высокая прогностическая значимость результатов таких широкодоступных инструментальных методов исследования, как ЭКГ покоя - наличие рубцовых изменений, тахикардии и нарушения ритма сердца, ЭхоКГ - нарушение локальной сократимости ЛЖ, расширение полостей желудочков сердца, наличие стенозов клапанов и диастолическая дисфункция, и ряда показателей ПДФН, что позволяет использовать их для оценки риска развития ССС у пациентов с ХИБС и определения показаний к проведению инвазивных диагностических процедур.
Gohlke HK. Identification of high-risk patients with coronary artery disease and good left ventricular function. Pract Cardiol 1988; 14 (1): 99-106.

8. The Task Force on Myocardial Revascularization of the European Society of Cardiology (ESC) and the European Association for Cardio-Thoracic Surgery (EACTS). Guidelines on myocardial revascularization. Eur Heart J 2010; 31: 2501-5. 9. Aronov DM, Lupanov VP. Atherosclerosis and coronary disease of heart. M.: Triad-X 2008; 248 p. Russian (Аронов Д. М., Лупанов В.П. Атеросклероз и коронарная болезнь сердца. М.: Триада-Х 2008; 248 c).

10. Hammermeister KE, DeRouen TA, Dodge HT. Variables predictive of survival in patients with coronary disease. Selection by univariate and multivariate analyses from the clinical, electrocardiographic, exercise, arteriographic, and quantitative angiographic evaluations. Circulation 1979; 59: 421-30.

11. DiazA, Bourassa MG, Guertin MC, TardifJC. Long-term prognostic value of resting heart rate in patients with suspected or proven coronary artery disease. Eur Heart J 2005; 26: 967-74.

12. Masur NA. Sudden death of patients with coronary heart disease. Moscow 1985 of prod. Medicine. 193 p. Russian (Мазур Н. А. Внезапная смерть больных ишемической болезнью сердца. Москва 1985 г. изд. Медицина. 193 с).

13. Lupanov VP. Depression of risk of complications and improvement of the quality of life at patients with chronic coronary heart disease. The Reference book of the polyclinic doctor 2009; 6: 15-9. Russian (Лупанов В.П. Снижение риска осложнений и улучшение прогноза жизни у больных хронической ишемической болезнью сердца. Справ. поликлин. врача 2009; 6: 15-9).

14. Dalya C, Norrieb J, Murdochc DL, et al. for the TIBET (Total Ischemic Burden European Trial) study group. The value of routine non-invasive tests to predict clinical outcome in stable angina. Eur Heart J 2003; 24: 532-40.

15. Massie BM, Carson JJ, McMurray, et al. Irbesartan in patients with heart failure and preserved ejection fraction. N Engl J Med 2008; 359 (23): 2456-67.

16. Lupanov VP. Prognostic indexes and predictors of electric instability of a myocardium at an exercise tolerance test at patients with coronary heart disease. Cardiovascular Therapy and Prevention 2006; 5: 10-2. Russian (Лупанов В.П. Прогностические индексы и предикторы электрической нестабильности миокарда при пробе с физической нагрузкой у больных ишемической болезнью сердца. Кардиоваскулярная терапия и профилактика 2006; 5: 10-2). 